QUARTERLY OF APPLIED MATHEMATICS

VOLUME LXIX, NUMBER 1

MARCH 2011, PAGES 57-78

S 0033-569X(2011)01203-2

Article electronically published on January 20, 2011

\title{
PRECISE BOUNDS FOR FINITE TIME BLOWUP OF SOLUTIONS TO VERY GENERAL ONE-SPACE-DIMENSIONAL NONLINEAR NEUMANN PROBLEMS
}

\author{
BY \\ KURT BRYAN (Department of Mathematics, Rose-Hulman Institute of Technology, Terre Haute, \\ Indiana 47803) \\ AND
}

MICHAEL S. VOGELIUS (Department of Mathematics, Rutgers University, New Brunswick, New Jersey 08903)

Abstract. In this paper we analyze the asymptotic finite time blowup of solutions to the heat equation with a nonlinear Neumann boundary flux in one space dimension. We perform a detailed examination of the nature of the blowup, which can occur only at the boundary, and we provide tight upper and lower bounds for the blowup rate for "arbitrary" nonlinear functions $F$, subject to very mild restrictions.

1. Introduction: the basic problem. Let $\Omega=(0,1) \subset \mathbb{R}$ and let $u(x, t)$ be a solution to the heat equation

$$
\frac{\partial u}{\partial t}-\frac{\partial^{2} u}{\partial x^{2}}=0, \quad 0<x<1, \quad 0<t<T,
$$

for some $T>0$, with the nonlinear Neumann boundary conditions

$$
\begin{aligned}
-\frac{\partial u}{\partial x}(0, t) & =F(u(0, t)), 0<t<T, \\
\frac{\partial u}{\partial x}(1, t) & =F(u(1, t)), 0<t<T,
\end{aligned}
$$

and initial condition

$$
u(x, 0)=f(x), 0<x<1 .
$$

Precise assumptions about the function $F$ will be stated later, but in general $F$ should be strictly increasing and grow superlinearly. There is a higher space-dimensional version of the problem in which $u(x, t)$ satisfies $\frac{\partial u}{\partial t}-\triangle u=0$ for $0<t<T$ and $x \in \Omega \subset \mathbb{R}^{N}$, $N \geq 2$, with Neumann boundary condition $\frac{\partial u}{\partial \mathbf{n}}=F(u)$ on $\partial \Omega$.

The solution $u(x, t)$ will frequently "blow up", that is, become unbounded, in finite time. In particular, it is well known, in any space dimension, that if the initial condition

Received June 2, 2009.

2000 Mathematics Subject Classification. Primary 35B05, 35B40, 45D05, 45G10, 45 M05.

Key words and phrases. Blowup, heat equation, nonlinear Neumann boundary condition. 
$f$ is of one sign, then blowup in finite time is assured (if the function $f$ changes sign, then the solution need not blow up; see for instance [2]). Early results on blowup for the heat equation with nonlinear boundary conditions were obtained in [9] and [13, where the authors demonstrate the inevitability of blowup for certain types of nonlinear boundary conditions and initial data, as well as for variations of the heat equation itself. Considerable work has been done on the problem (1)-(4) to determine, for example, where in $\bar{\Omega}$ the function $u$ will blow up (in general, on some subset of $\partial \Omega$ ), to provide upper and lower bounds on the time at which blowup will occur, and to provide upper and lower bounds for the solution near blowup. Most of this work has been done for nonlinear functions $F$ of rather specific forms, typically $F(u)=u^{p}$ for $p>1$ or $F(u)=e^{u}$. Many variations of the above basic model have also been examined. The articles [1, 5, 8 , provide a more complete survey of results concerning the blowup of solutions to the heat equation with these types of nonlinear boundary conditions.

In many papers, for example [4, 6, 7, 10, 11, special attention is given to the problem in one space dimension; see also [3]. In this case, equations (11)-(4) can be distilled down to a pair of, or even a single, nonlinear integral equation(s). Results from the theory of nonlinear integral equations can thus be of use. In particular, [12 provides a nice survey of recent results for these types of integral equations. However, sharp results on the asymptotic growth of the solution near blowup - whether for the heat equation or the integral equation formulation - exist only for the power law case $F(u)=u^{p}$, the exponential case $F(u)=e^{u}$, or simple variations of these.

As already indicated, the present paper considers only the one-space-dimensional case. In the first part, Section 3, we present a simple proof of the well-known fact that initial data of one sign leads to finite time blowup, and we provide an upper bound on the time at which blowup must occur. In Section 4 and subsections we establish quite sharp upper and lower bounds on the growth (in time) of solutions near blowup. The bounds are in terms of the functions $F(u) / u$ and $F^{\prime}(u)$, or rather, their inverses. Our upper and lower bounds for the solution behavior near blowup are new as far as the generality of the $F$ 's is concerned. For $F$ of polynomial or exponential type our bounds coincide with already known bounds.

2. Assumptions about $F$ and an integral equation formulation. We begin by making some very general assumptions about $F$, namely that

$$
F \in C^{2}(\mathbb{R}), F \text { is odd }(F(-u)=-F(u)), F^{\prime}(0)=1 \text {, and } F^{\prime \prime}(u)>0 \text { for } u>0 \text {. (A1) }
$$

Note that the last assumption implies that $F^{\prime}(u)$ is strictly increasing for $u>0$. The assumption that $F^{\prime}(0)=1$ is for convenience; we really need only that $F^{\prime}(0)>0$. By the odd symmetry we have $F(0)=0$. We shall also require that $F$ grow superlinearly in the sense that

$$
u \frac{F^{\prime}(u)}{F(u)} \geq 1+\delta_{1}, \quad u \geq M,
$$

for some positive constants $M$ and $\delta_{1}$. We note that

$$
\frac{d}{d u}\left(\frac{F(u)}{u^{1+\delta}}\right)=u^{\delta} F(u) \frac{u \frac{F^{\prime}(u)}{F(u)}-(1+\delta)}{u^{2+2 \delta}},
$$


and so according to assumption (A2) (and the fact that $F$ is odd, with $F(u)>0, u>0$ ) it follows that

$$
\begin{aligned}
& \frac{F(u)}{|u|^{1+\delta}},|u| \geq M, \quad \text { is strictly increasing for any } \delta<\delta_{1}, \\
& \text { and nondecreasing for } \delta=\delta_{1} .
\end{aligned}
$$

The facts that $F(0)=0$ and $F^{\prime \prime}(u)>0$ for $u>0$ are easily seen to imply that $F(u)<$ $u F^{\prime}(u)$ when $u>0$, and therefore

$$
\frac{F(u)}{|u|} \text { is strictly increasing on all of } \mathbb{R} \text {. }
$$

From (5) and (6) it follows that

$$
\frac{|F(u)|}{|u|^{1+\delta_{1}}} \geq c>0 .
$$

The facts that $F(0)=0$ and $F^{\prime}(0)=1$ ensure that the quantity $|F(u)| /|u|^{1+\delta_{1}}$ tends to $\infty$ as $u$ limits to 0 . We note that the strict monotonicity of $F^{\prime}(u)$ and $F(u) / u$ for $u>0$ imply the existence of well-defined (positive-valued) inverses for these functions, say, for arguments larger than 1.

Define $u_{0}(t):=u(0, t)$ and $u_{1}(t):=u(1, t)$. A standard argument involving the fundamental solution to the heat equation and integration by parts shows that (provided $u$ is sufficiently regular) the pair $\left(u_{0}(t), u_{1}(t)\right)$ satisfy the coupled nonlinear integral equations

$$
\begin{aligned}
u_{0}(t)= & \int_{0}^{t} K_{1}(t-s) u_{1}(s) d s+\int_{0}^{t} K_{3}(t-s) F\left(u_{1}(s)\right) d s \\
& +\int_{0}^{t} K_{2}(t-s) F\left(u_{0}(s)\right) d s+\frac{1}{\sqrt{\pi t}} \int_{0}^{1} e^{-\frac{x^{2}}{4 t}} f(x) d x, \\
u_{1}(t)= & \int_{0}^{t} K_{1}(t-s) u_{0}(s) d s+\int_{0}^{t} K_{3}(t-s) F\left(u_{0}(s)\right) d s \\
& +\int_{0}^{t} K_{2}(t-s) F\left(u_{1}(s)\right) d s+\frac{1}{\sqrt{\pi t}} \int_{0}^{1} e^{-\frac{(x-1)^{2}}{4 t}} f(x) d x,
\end{aligned}
$$

where

$$
K_{1}(t)=\frac{e^{-\frac{1}{4 t}}}{2 \sqrt{\pi} t^{3 / 2}}, \quad K_{2}(t)=\frac{1}{\sqrt{\pi t}}, \quad K_{3}(t)=\frac{e^{-\frac{1}{4 t}}}{\sqrt{\pi t}} .
$$

The solution $u(x, t)$ to the initial-boundary value problem (11)-(4) is then given by

$$
\begin{aligned}
u(x, t)= & \frac{1}{2 \sqrt{\pi t}} \int_{0}^{1} e^{-\frac{(x-y)^{2}}{4 t}} f(y) d y+\frac{1}{2} \int_{0}^{t} \frac{e^{-\frac{(x-1)^{2}}{4(t-s)}}}{\sqrt{\pi(t-s)}} F\left(u_{1}(s)\right) d s \\
& +\frac{1}{2} \int_{0}^{t} \frac{e^{-\frac{x^{2}}{4(t-s)}}}{\sqrt{\pi(t-s)}} F\left(u_{0}(s)\right) d s+x \int_{0}^{t} \frac{e^{-\frac{x^{2}}{4(t-s)}}}{4 \sqrt{\pi}(t-s)^{3 / 2}} u_{0}(s) d s \\
& +(1-x) \int_{0}^{t} \frac{e^{-\frac{(x-1)^{2}}{4(t-s)}}}{4 \sqrt{\pi}(t-s)^{3 / 2}} u_{1}(s) d s
\end{aligned}
$$

for $0<x<1$ and $0<t<T$. A standard contraction mapping argument shows that for any $f \in C^{0}[0,1]$ the equations (8) $-(9)$ possess a unique continuous solution on an 
interval $[0, T)$, for some $T>0$, and in fact both $u_{0}$ and $u_{1}$ belong to $C^{\alpha}[0, T)$ for any $\alpha<1$. If $f$ is nonnegative, then $u_{0}, u_{1}$, and $u(x, t)$ are nonnegative; indeed, if $f(x) \geq 0$ and $f$ is not identically zero, a simple maximum principle argument applied to (11)-(4) shows that for any fixed time $t_{0}>0$ we have $u\left(x, t_{0}\right) \geq f_{0}>0$ for some constant $f_{0}$ and all $x \in[0,1]$ (provided $u\left(x, t_{0}\right)$ exists).

3. Upper bound on the blowup time. Let $H(u)$ be an anti-derivative for $1 / F(u)$ for $u>0$. From the assumptions (A1) and (A2) (in particular, the consequence (5)) it's easy to see that $H(u)$ is strictly increasing and has a finite limit as $u \rightarrow \infty$. By addition of a suitable constant we may assume that $\lim _{u \rightarrow \infty} H(u)=0$. The function $H$ is invertible, with $H(u)<0$ for $u>0$. Also, since $F(0)=0$ and $F^{\prime}(0)=1$ we find that

$$
H(u)=\ln (u)+O(1)
$$

for $u$ near 0 . The inverse function, $H^{-1}$, is strictly increasing with $\lim _{z \rightarrow 0^{-}} H^{-1}(z)=\infty$.

Let "erf" denote the error function

$$
\operatorname{erf}(z)=\frac{2}{\sqrt{\pi}} \int_{0}^{z} e^{-x^{2}} d x, z>0
$$

and let $f_{0}$ be a fixed positive number.

We note that $\operatorname{erf}(z)=\frac{2 z}{\sqrt{\pi}}+O\left(z^{3}\right)$ for $z$ near zero, and therefore $\operatorname{erf}(1 /(2 \sqrt{t}))$ behaves asymptotically like $1 / \sqrt{\pi t}$ as $t \rightarrow \infty$. As a consequence of this and (12) it now follows that

$$
-\sqrt{\pi t} H\left(f_{0} \operatorname{erf}(1 /(2 \sqrt{t}))\right) \text { behaves asymptotically like } \frac{\sqrt{\pi t}}{2} \ln t,
$$

as $t \rightarrow \infty$. From the fact that $\operatorname{erf}(z)$ limits to 1 as $z \rightarrow \infty$ we also easily see that

$$
-\sqrt{\pi t} H\left(f_{0} \operatorname{erf}(1 /(2 \sqrt{t}))\right) \text { behaves asymptotically like }-H\left(f_{0}\right) \sqrt{\pi t},
$$

as $t \rightarrow 0_{+}$. Consequently the equation

$$
t=-\sqrt{\pi t} H\left(f_{0} \operatorname{erf}(1 /(2 \sqrt{t}))\right)
$$

has at least one positive solution, and indeed it has a smallest positive solution $t_{1}$. We proceed to establish the following result.

Proposition 3.1. Let $F$ satisfy assumptions (A1) and (A2). Suppose $f(x) \geq f_{0}$ for some positive constant $f_{0}$, and let $u_{0}, u_{1}$ denote the solutions to (8)-(9). Let $t_{1}$ denote the smallest positive solution to (13). Then there exists $0<t^{*} \leq t_{1}$ such that

(1) $u_{0}$ and $u_{1}$ are both in $C^{\alpha}\left[0, t^{*}\right)$ for some $\alpha>1 / 2$,

(2) at least one of the functions $u_{0}$ and $u_{1}$ fails to be bounded as $t$ approaches $t^{*}$.

Proof. The contraction mapping argument referred to in the previous section establishes the existence of continuous solutions $u_{0}$ and $u_{1}$ on some interval $[0, t)$ with $t>0$. Moreover, $u_{0}$ and $u_{1}$ must belong to $C^{\alpha}[0, t)$ for any $\alpha<1$ on such an interval $[0, t)$. Define

$$
T=\sup \left\{t>0: u_{i} \in C^{\alpha}[0, t) \cap L^{\infty}(0, t)\right\} .
$$

If $T$ is finite, then at least one of these two functions must fail to be in $L^{\infty}(0, T)$ (otherwise the contraction mapping argument yields existence beyond $T$ ). There are potentially two 
possibilities: (1) $T \leq t_{1}$, or (2) $T>t_{1}$. In the first scenario we know that at least one of the functions $u_{0}$ and $u_{1}$ is in $L^{\infty}(0, t)$ for any $t<T$ but not in $L^{\infty}(0, T)$. This partially verifies the proposition with $t^{*}=T \leq t_{1}$. We now proceed to show that the second scenario cannot occur, thus completing the proof of the proposition.

Let's focus on the left end of the domain $(x=0)$ and the function $u_{0}(t)$, and suppose we are in the second scenario. Consider equation (8). Since the functions $u_{0}$ and $u_{1}$ are positive and bounded we see that the integrals involving $u_{1}$ are positive and finite for any $t<T$. As a result we can drop these terms to obtain

$$
u_{0}(t) \geq \int_{0}^{t} K_{2}(t-s) F\left(u_{0}(s)\right) d s+\frac{1}{\sqrt{\pi t}} \int_{0}^{1} e^{-\frac{x^{2}}{4 t}} f(x) d x
$$

for $0<t<T$; the function $u_{1}$ has completely dropped out. We shall show that the above inequality alone implies that $u_{0}$ blows up at $t_{1}$ at the latest, which is a contradiction to the basic assumption of the second scenario $\left(T>t_{1}\right)$. This will complete the proof of Proposition 3.1. Using the monotonicity of the error function we can bound

$$
\frac{1}{\sqrt{\pi t}} \int_{0}^{1} e^{-\frac{x^{2}}{4 t}} f(x) d x \geq \frac{f_{0}}{\sqrt{\pi t}} \int_{0}^{1} e^{-\frac{x^{2}}{4 t}} d x=f_{0} \cdot \operatorname{erf}\left(\frac{1}{2 \sqrt{t}}\right)>f_{0} \cdot \operatorname{erf}\left(\frac{1}{2 \sqrt{t_{1}}}\right)
$$

for $0 \leq t<t_{1}$. From (14) we then have

$$
u_{0}(t)>\int_{0}^{t} K_{2}(t-s) F\left(u_{0}(s)\right) d s+f_{0} \cdot \operatorname{erf}\left(\frac{1}{2 \sqrt{t_{1}}}\right)
$$

for $0 \leq t<t_{1}$. After insertion of the formula (10) for $K_{2}$, this yields

$$
\begin{aligned}
u_{0}(t) & >\frac{1}{\sqrt{\pi}} \int_{0}^{t} \frac{F\left(u_{0}(s)\right)}{\sqrt{t-s}} d s+f_{0} \cdot \operatorname{erf}\left(\frac{1}{2 \sqrt{t_{1}}}\right) \\
& \geq \frac{1}{\sqrt{\pi t}} \int_{0}^{t} F\left(u_{0}(s)\right) d s+f_{0} \cdot \operatorname{erf}\left(\frac{1}{2 \sqrt{t_{1}}}\right) \\
& \geq \frac{1}{\sqrt{\pi t_{1}}} \int_{0}^{t} F\left(u_{0}(s)\right) d s+f_{0} \cdot \operatorname{erf}\left(\frac{1}{2 \sqrt{t_{1}}}\right)
\end{aligned}
$$

for $0 \leq t<t_{1}$. Let

$$
v(s)=\int_{0}^{s} F\left(u_{0}(t)\right) d t
$$

so that $v^{\prime}(s)=F\left(u_{0}(s)\right)$ and $u_{0}(s)=F^{-1}\left(v^{\prime}(s)\right)$. The estimate (15) now reads

$$
F^{-1}\left(v^{\prime}(s)\right)>\frac{1}{\sqrt{\pi t_{1}}} v(s)+f_{0} \cdot \operatorname{erf}\left(\frac{1}{2 \sqrt{t_{1}}}\right), 0 \leq s<t_{1} .
$$

Since $F$ is strictly increasing, this implies that $v^{\prime}(s)>F\left[v(s) / \sqrt{\pi t_{1}}+f_{0} \cdot \operatorname{erf}\left(1 /\left(2 \sqrt{t_{1}}\right)\right)\right]$, which we may rewrite as

$$
\frac{v^{\prime}(s)}{F\left[v(s) / \sqrt{\pi t_{1}}+f_{0} \cdot \operatorname{erf}\left(1 /\left(2 \sqrt{t_{1}}\right)\right)\right]}>1,0 \leq s<t_{1} .
$$

As before, let $H(u)<0$ be the anti-derivative of $1 / F(u), u>0$, satisfying $\lim _{u \rightarrow \infty} H(u)$ $=0$. Since $v(0)=0$, integration of the inequality (16) from 0 to $t<t_{1}$ yields

$$
\sqrt{\pi t_{1}} H\left[\frac{v(t)}{\sqrt{\pi t_{1}}}+f_{0} \cdot \operatorname{erf}\left(\frac{1}{2 \sqrt{t_{1}}}\right)\right]-\sqrt{\pi t_{1}} H\left[f_{0} \cdot \operatorname{erf}\left(\frac{1}{2 \sqrt{t_{1}}}\right)\right]>t,
$$


so that

$$
H\left[\frac{v(t)}{\sqrt{\pi t_{1}}}+f_{0} \cdot \operatorname{erf}\left(\frac{1}{2 \sqrt{t_{1}}}\right)\right]>\frac{t}{\sqrt{t_{1} \pi}}+H\left[f_{0} \cdot \operatorname{erf}\left(\frac{1}{2 \sqrt{t_{1}}}\right)\right] .
$$

Note that the right-hand side of (17) must be negative and thus lies in the domain of definition of $H^{-1}$. We now apply $H^{-1}$ (strictly increasing) to both sides of (17) to arrive at

$$
v(t)>\left[H^{-1}\left[\frac{t}{\sqrt{t_{1} \pi}}+H\left(f_{0} \cdot \operatorname{erf}\left(\frac{1}{2 \sqrt{t_{1}}}\right)\right)\right]-f_{0} \cdot \operatorname{erf}\left(\frac{1}{2 \sqrt{t_{1}}}\right)\right] \sqrt{\pi t_{1}} .
$$

As $t<t_{1}$ approaches $t_{1}$, the expression

$$
\frac{t}{\sqrt{t_{1} \pi}}+H\left(f_{0} \cdot \operatorname{erf}\left(\frac{1}{2 \sqrt{t_{1}}}\right)\right)
$$

approaches

$$
\sqrt{\frac{t_{1}}{\pi}}+H\left(f_{0} \cdot \operatorname{erf}\left(\frac{1}{2 \sqrt{t_{1}}}\right)\right)=0 .
$$

Since $H^{-1}(z)$ tends to $\infty$ as $z \rightarrow 0^{-}$, it follows that

$$
H^{-1}\left[\frac{t}{\sqrt{t_{1} \pi}}+H\left(f_{0} \cdot \operatorname{erf}\left(\frac{1}{2 \sqrt{t_{1}}}\right)\right)\right] \rightarrow \infty, \text { as } t \rightarrow t_{1} \text { from below. }
$$

The inequality (18) implies that $v(t)$ becomes unbounded as $t$ approaches $t_{1}$ from below, and so $u_{0}(t)$ also becomes unbounded as $t$ approaches $t_{1}$ from below. Indeed, a direct combination of (15) and (18) yields the following lower bound for $u_{0}(t)$ :

$$
\begin{aligned}
u_{0}(t) & >\frac{1}{\sqrt{\pi t_{1}}} v(t)+f_{0} \cdot \operatorname{erf}\left(\frac{1}{2 \sqrt{t_{1}}}\right) \\
& >H^{-1}\left[\frac{t}{\sqrt{t_{1} \pi}}+H\left(f_{0} \cdot \operatorname{erf}\left(\frac{1}{2 \sqrt{t_{1}}}\right)\right)\right], 0 \leq t<t_{1} .
\end{aligned}
$$

This establishes the impossibility of the second scenario $\left(T>t_{1}\right)$ and completes the proof of the proposition.

REMARK 1. In Proposition 3.1 we can relax the condition $f(x) \geq f_{0}$ to $f(x) \geq 0$ with $f$ not identically 0 . To see this simply note that if $f(x) \geq 0$ and is not identically 0 , then the maximum principle guarantees that $u(x, \epsilon) \geq f_{\epsilon}$, where $f_{\epsilon}$ is a positive constant, for some fixed $\epsilon>0$. We may now apply Proposition 3.1 with $\epsilon$ as the initial time. $t_{1}$ thus gets replaced by $t_{1}+\epsilon$, where $t_{1}$ is the smallest positive solution to $t=$ $-\sqrt{\pi t} H\left(f_{\epsilon} \cdot \operatorname{erf}(1 /(2 \sqrt{t}))\right)$. Proposition 3.1 also holds if $f(x) \leq 0$ with $f$ not identically 0 . To prove this we simply replace $u$ by $-u$, and note that, due to the odd symmetry of $F$, this function solves the same initial boundary value problem as $u$ only with $f$ replaced by $-f$.

REMARK 2. As noted in the introduction, if the initial condition $f$ changes sign, then blowup need not occur. In [2] we show that sign-changing solutions with certain symmetries may in fact decay to zero if $f$ is "small enough", while other solutions with the same symmetries must blow up in finite time. 
4. Asymptotic bounds near blowup. In the following two sections we provide a more detailed study of the asymptotic structure of solutions to equations (11)-(4) near the time of blowup. The nonlinearity $F$ is of a very general superlinear nature, with the very mild assumptions (A1) and (A2) described in Section 2. From the representation formula (11) for $u(x, t)$ in terms of the initial data and $u(0, t), u(1, t)$, it is clear that blowup at time $t^{*}$ always implies blowup at one or both boundary points. As we shall see later (after establishing an upper bound for endpoint blowup) the solution always stays bounded in the interior, even when boundary blowup occurs. In general, blowup will happen at one boundary point, and the solution will remain bounded at the other.

In the remainder of this section we shall assume that $u_{0}(t):=u(0, t)$ and $u_{1}(t):=$ $u(1, t)$ are sufficiently smooth and defined on $\left[0, t^{*}\right)$, that $u_{1}$ becomes unbounded near $t^{*}<\infty$, but that $u_{0}$ remains bounded (the pathological case in which blowup appears simultaneously at both endpoints requires a slightly different analysis which we do not present here). For simplicity we assume that $u_{1}$ attains arbitrarily large positive values as $t$ approaches $t^{*}$. From the integral formulation (9) we know that $u_{1} \in C^{\alpha}\left[0, t^{*}\right)$ is a solution to

$$
u_{1}(t)=\frac{1}{\sqrt{\pi}} \int_{0}^{t} \frac{F\left(u_{1}(s)\right)}{\sqrt{t-s}} d s+q(t)
$$

where $q$ is a $C^{1}$ function on the closed interval $\left[0, t^{*}\right]$. In Appendix $\mathrm{A}$ we prove the following monotonicity result.

Proposition 4.1. Let $\phi(t)$ be a function in $C^{\alpha}\left[0, t^{*}\right)$ for some $\alpha>1 / 2,0<t^{*}<\infty$, and suppose $\phi$ satisfies an integral equation of the form

$$
P(\phi(t))=\int_{0}^{t} \frac{H(s, \phi(s))}{\sqrt{t-s}} d s+q(t),
$$

where $P \in C^{1}(-\infty, \infty)$ is strictly increasing, $q \in C^{1}\left[0, t^{*}\right]$, and $H \in C^{1}\left(\left[0, t^{*}\right) \times\right.$ $(-\infty, \infty))$. Assume that $H(s, z)$ is nondecreasing in each argument with

$$
\lim _{z \rightarrow \infty} H(s, z)=\infty
$$

for each fixed $s \in\left[0, t^{*}\right)$, and assume that

$$
\limsup _{t \rightarrow t^{*}} \phi(t)=\infty .
$$

For $R \in(\inf \phi, \infty)$ let $t_{R} \in\left[0, t^{*}\right)$ be defined as

$$
t_{R}=\inf \left\{t \in\left[0, t^{*}\right): \phi(t)=R\right\} .
$$

Then there exists $R$ such that $\phi(t)$ is strictly increasing for $t_{R} \leq t<t^{*}$.

REMARK 3. Proposition 4.1, in combination with the fact that $u_{1}$ attains arbitrarily large positive values as $t$ approaches $t^{*}$, shows that $u_{1}$ is strictly increasing in some interval $\left(t_{1}, t^{*}\right)$. By changing the initial time, if necessary, we may without loss of generality assume that $u_{1}$ is positive and strictly increasing on the whole interval $\left(0, t^{*}\right)$. 
We now proceed to establish upper and lower bounds for the blowup of behavior of $u_{1}$ near $t^{*}$. In brief, we shall prove that

$$
K_{1}\left(F^{\prime}\right)^{-1}\left(C_{1} / \sqrt{t^{*}-t}\right) \leq u_{1}(t) \leq K_{2} G^{-1}\left(C_{2} /\left(\sqrt{t^{*}-t}\right)^{\gamma}\right)
$$

for constants $K_{i}$ and $C_{i}$, and $\gamma$ larger than, but arbitrarily close to 1 . The function $G$ is defined as $G(u)=F(u) / u$. Note that as part of the assumption (A1), $F(0)=0$ and $F^{\prime \prime}(u)>0$ for $u>0$. It follows immediately that $G \leq F^{\prime}$, and therefore due to the strict monotonicity of $F^{\prime}$ and $G$ (both are increasing for positive arguments) we obtain $\left(F^{\prime}\right)^{-1} \leq G^{-1}$ for positive arguments. However, let us note that the bounds (20) are still true even when $F(0) \neq 0$ and provided only that $F(u), F^{\prime}(u)$, and $F^{\prime \prime}(u)$ are positive for $u$ sufficiently large; see Remark 6. We suspect that the presence of $\gamma>1$ in the upper bound is a technical artifact, but we are presently unable to derive the bound with $\gamma=1$.

4.1. Lower bound for growth.

Proposition 4.2. Let $F$ satisfy assumptions (A1) and (A2). Let $u_{0}(t)$ and $u_{1}(t)$ be $C^{\alpha}\left[0, t^{*}\right)$ solutions to equations (8) (9) for some $0<t^{*}<\infty$. Suppose that $u_{0}(t)$ remains bounded and that $u_{1}(t)$ attains arbitrarily large positive values for $t$ near $t^{*}$. Let $K^{*}$ denote the constant $K^{*}=1-\limsup _{z \rightarrow \infty} \frac{F(z)}{z F^{\prime}(z)}>0$, and let $0<K<K^{*}$, and $0<C<\sqrt{\pi} / 2$. Then

$$
u_{1}(t) \geq K\left(F^{\prime}\right)^{-1}\left(C / \sqrt{t^{*}-t}\right)
$$

for $t$ near $t^{*}$.

Proof. As discussed in Remark 3, we may without loss of generality assume that $u_{1}(t)$ is positive and strictly increasing for $0<t<t^{*}$. For $0<t<t+h<t^{*}$ we have from equation (19),

$$
\begin{aligned}
u_{1}(t+h) & =\frac{1}{\sqrt{\pi}} \int_{0}^{t+h} \frac{F\left(u_{1}(s)\right)}{\sqrt{t+h-s}} d s+q(t+h) \\
& =\frac{1}{\sqrt{\pi}} \int_{0}^{t} \frac{F\left(u_{1}(s)\right)}{\sqrt{t+h-s}} d s+\frac{1}{\sqrt{\pi}} \int_{t}^{t+h} \frac{F\left(u_{1}(s)\right)}{\sqrt{t+h-s}} d s+q(t+h) \\
& \leq \frac{1}{\sqrt{\pi}} \int_{0}^{t} \frac{F\left(u_{1}(s)\right)}{\sqrt{t-s}} d s+F\left(u_{1}(t+h)\right) \frac{1}{\sqrt{\pi}} \int_{t}^{t+h} \frac{1}{\sqrt{t+h-s}} d s+q(t+h) \\
& =u_{1}(t)+2 \frac{\sqrt{h}}{\sqrt{\pi}} F\left(u_{1}(t+h)\right)+q(t+h)-q(t)
\end{aligned}
$$

where the inequality follows from $0 \leq F\left(u_{1}(s)\right) \leq F\left(u_{1}(t+h)\right)$ for $0 \leq s \leq t+h$. We now use the fact that $|q(t+h)-q(t)| \leq C_{q} h$ to obtain

$$
u_{1}(t+h)-2 \frac{\sqrt{h}}{\sqrt{\pi}} F\left(u_{1}(t+h)\right) \leq u_{1}(t)+C_{q} h,
$$

for $0<t<t+h<t^{*}$. Note that since $q \in C^{1}\left[0, t^{*}\right]$ we can choose $C_{q}$ independently of $t$ and $h$. Let $G_{h}$ denote the function

$$
G_{h}(x)=x-2 \frac{\sqrt{h}}{\sqrt{\pi}} F(x) .
$$


For a given $z>u_{1}(t)$ consider that (unique) $h$ such that $u_{1}(t+h)=z$. Equation (21) shows that

$$
G_{h}(z) \leq u_{1}(t)+C_{q} h .
$$

The above inequality leads to a lower bound for $h$ (e.g., when $h=0$ the inequality is violated, for it becomes $\left.z \leq u_{1}(t)\right)$. For fixed $z>u_{1}(t)$ the quantity $G_{h}(z)$ is strictly decreasing in $h$, and limits to $-\infty$ as $h$ increases, so there is a unique $h_{z}>0$ for which

$$
G_{h_{z}}(z)=u_{1}(t)+C_{q} h_{z} .
$$

This $h_{z}$ provides a lower bound for the value of $h$ for which $u_{1}(t+h)=z$ : in other words, $h_{z} \leq h$. We can solve equation (22) for $h_{z}$ to arrive at

$$
h_{z}=\frac{F^{2}(z)}{C_{q}^{2} \pi}(2+p-2 \sqrt{1+p})
$$

with $p=\frac{C_{q} \pi\left(z-u_{1}(t)\right)}{F^{2}(z)}$. A simple (but asymptotically sharp) lower bound on $h_{z}$ can be obtained from the fact that $2+p-2 \sqrt{1+p} \geq \frac{p^{2}}{2(p+2)}$ for all $p \geq 0$. We obtain

$$
\begin{aligned}
h_{z} & \geq \frac{F^{2}(z)}{C_{q}^{2} \pi} \frac{p^{2}}{2(p+2)} \\
& =\frac{\pi\left(z-u_{1}(t)\right)^{2}}{4 F^{2}(z)+2 C_{q} \pi\left(z-u_{1}(t)\right)} .
\end{aligned}
$$

Due to the superlinearity of $F$ we obtain, for any $C_{0}<1$ (and sufficiently large values of $\left.u_{1}(t)\right)$ that

$$
h \geq h_{z} \geq C_{0} \frac{\pi\left(z-u_{1}(t)\right)^{2}}{4 F^{2}(z)}
$$

for any $z \geq u_{1}(t)$, where $u_{1}(t+h)=z$. The constant $C_{0}$ is independent of $t$ and $h$.

Let $v=u_{1}^{-1}$, which is well-defined, since $u_{1}$ is strictly increasing. Using the notation $u_{1}(t)=z_{1}$ we have $v(z)-v\left(z_{1}\right)=h$, and the inequality (23) may be written as

$$
v(z)-v\left(z_{1}\right) \geq C_{1} \frac{\left(z-z_{1}\right)^{2}}{F^{2}(z)}
$$

with $C_{1}=\frac{C_{0} \pi}{4}$. This inequality holds for any sufficiently large $z_{1}$ and all $z \geq z_{1}$. We know that $u_{1}$ blows up monotonically as $t \rightarrow t^{*}$, and so $v$ is strictly increasing, with

$$
\lim _{z \rightarrow \infty} v(z)=t^{*} .
$$

For $\epsilon>0$ sufficiently small we now choose $z_{1}=u_{1}\left(t^{*}-\epsilon\right)$ so that $v\left(z_{1}\right)=t^{*}-\epsilon$. Since $v(z)<t^{*}, z_{1} \leq z$, the estimate (24) now yields

$$
C_{1} \frac{\left(z-z_{1}\right)^{2}}{F^{2}(z)} \leq v(z)-v\left(z_{1}\right)<\epsilon
$$

for all $z \geq z_{1}$. We get as much out of the estimate (25) as possible by taking $z \geq z_{1}$, which maximizes the left-hand side. It's easy to see that there is a unique such $z$, for the left side of (25) equals 0 when $z=z_{1}$, tends to zero as $z \rightarrow \infty$, and has a stationary point $z=z_{1}^{*}$ that satisfies

$$
z_{1}^{*}-\frac{F\left(z_{1}^{*}\right)}{F^{\prime}\left(z_{1}^{*}\right)}=z_{1}
$$


easily found by differentiating the left side of (25). That the equation (26) has a unique solution $z_{1}^{*}>z_{1}$ is a consequence of the fact that the function $z-\frac{F(z)}{F^{\prime}(z)}, z_{1} \leq z$, is strictly increasing (a consequence of $F^{\prime \prime}>0$ ) with

$$
z_{1}-\frac{F\left(z_{1}\right)}{F^{\prime}\left(z_{1}\right)}<z_{1} \text { and } \lim _{z \rightarrow \infty} z-\frac{F(z)}{F^{\prime}(z)}=\infty .
$$

The latter assertion is a consequence of assumption (A2), which implies that

$$
\frac{F(z)}{z F^{\prime}(z)} \leq \frac{1}{1+\delta_{2}}<1
$$

for some $\delta_{2}>0$, and so

$$
z-\frac{F(z)}{F^{\prime}(z)}=z\left(1-\frac{F(z)}{z F^{\prime}(z)}\right) \rightarrow \infty \text { as } z \rightarrow \infty .
$$

From (27) and (28) it furthermore follows that with $K^{*}$ as defined in the statement of the proposition,

$$
K^{*}=1-\limsup _{z \rightarrow \infty} \frac{F(z)}{z F^{\prime}(z)} \geq \frac{\delta_{2}}{1+\delta_{2}}>0,
$$

and that, for any $K<K^{*}$,

$$
K z_{1}^{*}<z_{1}^{*}-\frac{F\left(z_{1}^{*}\right)}{F^{\prime}\left(z_{1}^{*}\right)}=z_{1}
$$

for $z_{1}$ sufficiently large. Of course this implies that

$$
z_{1}^{*} \leq \frac{z_{1}}{K},
$$

for $z_{1}$ sufficiently large. If we use $z=z_{1}^{*}$ in (25), then (26) gives

$$
C_{1} \frac{1}{\left(F^{\prime}\left(z_{1}^{*}\right)\right)^{2}} \leq \epsilon
$$

which in combination with (29), and the fact that $F^{\prime}$ is increasing, yields

$$
C_{1} \frac{1}{\left(F^{\prime}\left(z_{1} / K\right)\right)^{2}} \leq \epsilon
$$

for $z_{1}$ sufficiently large. We conclude that, with $C=\sqrt{C_{1}}$,

$$
z_{1} \geq K\left(F^{\prime}\right)^{-1}(C / \sqrt{\epsilon}),
$$

for $z_{1}$ sufficiently large. Inequality (30) yields a lower bound on the growth of $u_{1}$ near $t^{*}$ if we note that $z_{1}=u_{1}\left(t^{*}-\epsilon\right)$, namely

$$
u_{1}\left(t^{*}-\epsilon\right) \geq K\left(F^{\prime}\right)^{-1}(C / \sqrt{\epsilon}), \text { for } \epsilon \text { sufficiently small }
$$

or

$$
u_{1}(t) \geq K\left(F^{\prime}\right)^{-1}\left(C / \sqrt{t^{*}-t}\right), \text { for } t \text { near } t^{*} .
$$

Here $C=\sqrt{C_{1}}=\sqrt{C_{0} \pi} / 2<\sqrt{\pi} / 2$ can be arbitrarily close to $\sqrt{\pi} / 2$, and $K<K^{*}$ can be arbitrarily close to $K^{*}$. This proves Proposition 4.2 . 
REMARK 4. To illustrate the above bound, let us consider a couple of typical examples. With $F(u)=\sinh (u)$ it's easy to check that $K^{*}=1$. In this case Proposition 4.2 yields a lower bound of the form

$$
u_{1}(t) \geq K \operatorname{arccosh}\left(C /\left(t^{*}-t\right)^{1 / 2}\right)=-\frac{K}{2} \ln \left(t^{*}-t\right)+O(1),
$$

for any $K<1$.

For $F(u)=|u|^{p-1} u+u$ with $p>1$, Proposition 4.2 gives

$$
u_{1}(t) \geq K_{p}\left(t^{*}-t\right)^{-1 /(2(p-1))},
$$

for some positive constant $K_{p}$, smaller than, but arbitrarily close to

$$
(\sqrt{\pi} / 2)^{1 /(p-1)} p^{-p /(p-1)}(p-1) .
$$

It is easy to check that $K_{p}$ must approach 0 as $p \rightarrow 1$, and that $K_{p}$ may be picked arbitrarily close to 1 as $p \rightarrow \infty$.

4.2. Upper bound for growth. In order to establish an upper bound on the solution near blowup we shall make one more assumption on the behavior of $F$, in addition to those of Section 2. We shall require that

There exists $M>0$ such that $F^{\prime}(u) / F(u)$ is nonincreasing for $u \geq M$.

This assumption and those of Section 2 are all satisfied, for example, by $F(u)=|u|^{p-1} u+$ $u, p>1$, and $F(u)=\sinh (u)$. Assumption (A3) implies that $F^{\prime}(u) / F(u) \leq K_{2}$ for $M \leq u$ and some constant $K_{2}$. Integration then yields $F(u) \leq C e^{K_{2} u}$ for $M \leq u$, and from continuity we obtain $F(u) \leq C_{2} e^{K_{2} u}$ for some constant $C_{2}$ and all $u>0$. In combination with (7) we conclude that our assumptions about $F$ imply the existence of constants $K_{1}>1$ and positive constants $K_{2}, C_{1}, C_{2}$ so that

$$
C_{1} u^{K_{1}} \leq F(u) \leq C_{2} e^{K_{2} u} \text { for all } u>0 .
$$

The a priori assumptions about the solution $u$ near $t^{*}$ are as in the previous section; that is, we assume that $u_{0}(t):=u(0, t)$ and $u_{1}(t):=u(1, t)$ are smooth and defined on $\left[0, t^{*}\right)$, that $u_{1}$ becomes unbounded near $t^{*}$, but that $u_{0}$ remains bounded. For simplicity we assume that $u_{1}$ attains arbitrarily large positive values as $t$ approaches $t^{*}$. Finally we recall that $u_{1} \in C^{\alpha}\left[0, t^{*}\right)$ (any $\alpha<1$ ) satisfies the integral equation (19) where $q$ is a $C^{1}$ function on the closed interval $\left[0, t^{*}\right]$.

Proposition 4.3. Let the assumptions be as in Proposition 4.2, and additionally suppose that (A3) holds. Then for any $\gamma>1$ and any $K>1$ there exists a constant $C$ such that

$$
u_{1}(t) \leq K G^{-1}\left(C /\left(\sqrt{t^{*}-t}\right)^{\gamma}\right)
$$

for $t$ near $t^{*}$. Here $G(u)=F(u) / u$.

Proof. As argued in Remark [3] we may without loss of generality assume that $u_{1}(t)$ is positive and strictly increasing for $0<t<t^{*}$. Let $b_{n}, n \geq 0$, be a sequence that limits to infinity, starting with $b_{0}>u_{1}(0)$. Indeed, let us suppose that we have $b_{n}=b(n)$ for some strictly increasing function $b(x)$ of a real variable $x$. Let $a_{n} \in\left(0, t^{*}\right)$ be chosen 
(uniquely) so that $u_{1}\left(a_{n}\right)=b_{n}$. Note that $0<a_{0}<a_{1}<\ldots<a_{n}<a_{n+1}<\ldots$ with $a_{n} \rightarrow t^{*}$, as $n \rightarrow \infty$. We then have

$$
\begin{aligned}
b_{n} & =u_{1}\left(a_{n}\right) \\
& =\int_{0}^{a_{n}} \frac{F\left(u_{1}(s)\right) d s}{\sqrt{a_{n}-s}}+q\left(a_{n}\right) \\
& =\sum_{k=0}^{n-1} \int_{a_{k}}^{a_{k+1}} \frac{F\left(u_{1}(s)\right) d s}{\sqrt{a_{n}-s}}+q\left(a_{n}\right) \\
& \geq \sum_{k=0}^{n-1} \int_{a_{k}}^{a_{k+1}} \frac{F\left(u_{1}\left(a_{k}\right)\right) d s}{\sqrt{a_{n}-s}}+q\left(a_{n}\right) \\
& =\sum_{k=0}^{n-1} \int_{a_{k}}^{a_{k+1}} \frac{F\left(b_{k}\right) d s}{\sqrt{a_{n}-s}}+q\left(a_{n}\right) \\
& =2 \sum_{k=0}^{n-1} F\left(b_{k}\right)\left(\sqrt{a_{n}-a_{k}}-\sqrt{a_{n}-a_{k+1}}\right)+q\left(a_{n}\right) \\
& \geq 2 F\left(b_{n-1}\right) \sqrt{a_{n}-a_{n-1}}-Q,
\end{aligned}
$$

where we use that $u$ is strictly increasing, and $q\left(a_{n}\right) \geq-Q$, and drop all but the $k=n-1$ term in the last sum. A little rearrangement of (31) yields

$$
a_{n}-a_{n-1} \leq \frac{\left(b_{n}+Q\right)^{2}}{4 F^{2}\left(b_{n-1}\right)},
$$

and a straightforward telescoping summation then shows that

$$
a_{n}-a_{m} \leq \frac{1}{4} \sum_{k=m+1}^{n} \frac{\left(b_{k}+Q\right)^{2}}{F^{2}\left(b_{k-1}\right)} .
$$

We let $n$ tend to infinity to find

$$
t^{*}-a_{m} \leq \frac{1}{4} \sum_{k=m+1}^{\infty} \frac{\left(b_{k}+Q\right)^{2}}{F^{2}\left(b_{k-1}\right)} .
$$

From now on we take $b_{k}=b_{0} d^{k}$ for some fixed $d>1$, and so, based on the previous estimate,

$$
\begin{aligned}
t^{*}-a_{m} & \leq \frac{1}{4} \sum_{k=m+1}^{\infty} \frac{\left(b_{k}+Q\right)^{2}}{F^{2}\left(b_{k-1}\right)} \\
& =\frac{1}{4} \sum_{k=m+1}^{\infty} \frac{\left(d b_{k-1}+Q\right)^{2}}{F^{2}\left(b_{k-1}\right)} \\
& =\frac{1}{4} \sum_{k=m}^{\infty} \frac{\left(d b_{k}+Q\right)^{2}}{F^{2}\left(b_{k}\right)}, m \geq 0 .
\end{aligned}
$$

Since $Q$ is fixed and $b_{k} \rightarrow \infty$, as $k \rightarrow \infty$, it follows that, given any $D>1$,

$$
t^{*}-a_{m} \leq \frac{d^{2} D}{4} \sum_{k=m}^{\infty} \frac{b_{k}^{2}}{F^{2}\left(b_{k}\right)}
$$


for $m$ sufficiently large. Define

$$
\phi(m)=\sum_{k=m}^{\infty} \frac{b_{k}^{2}}{F^{2}\left(b_{k}\right)}=b_{0}^{2} \sum_{k=m}^{\infty} \frac{d^{2 k}}{F^{2}\left(b_{0} d^{k}\right)} .
$$

Note that $\phi>0$, and that $\phi$ is strictly decreasing in $m$, with $\phi(m) \rightarrow 0$ as $m \rightarrow \infty$. Of course, $\phi$ is defined only on the nonnegative integers, but we may extend $\phi$ to a function with the same properties on the nonnegative reals. From (32) we have

$$
t^{*}-a_{m} \leq \frac{d^{2} D}{4} \phi(m)=\frac{d^{2} D}{4} \phi\left(b^{-1}\left(u\left(a_{m}\right)\right)\right),
$$

since $b^{-1}\left(u\left(a_{m}\right)\right)=b^{-1}\left(b_{m}\right)=m$. Divide both sides above by $d^{2} D / 4$ and apply the strictly decreasing function $\psi=b \circ \phi^{-1}$ to both sides (which reverses the inequality) to find

$$
u\left(a_{m}\right) \leq \psi\left(\frac{4}{d^{2} D}\left(t^{*}-a_{m}\right)\right)
$$

for $m$ sufficiently large. We note that $\psi(s) \rightarrow \infty$ as $s \rightarrow 0^{+}$. Given $\epsilon$ sufficiently small we have $t^{*}-\epsilon \in\left[a_{m-1}, a_{m}\right)$ for some $m \geq 1$ (with $m \rightarrow \infty$ as $\epsilon \rightarrow 0$ ). The estimate (34) then yields (recall $u\left(a_{m}\right)=b_{m}$ )

$$
\begin{aligned}
u\left(t^{*}-\epsilon\right) \leq u\left(a_{m}\right) & =\frac{b_{m}}{b_{m-1}} u\left(a_{m-1}\right) \\
& \leq d \psi\left(\frac{4}{d^{2} D}\left(t^{*}-a_{m-1}\right)\right) \\
& \leq d \psi\left(\frac{4 \epsilon}{d^{2} D}\right),
\end{aligned}
$$

for $m$ sufficiently large, or $\epsilon$ sufficiently small. Here we can take any $D>1$ (though $D$ closer to 1 may require smaller $\epsilon$ ). It only remains to bound $\psi$. Comparison to an integral shows that, with $\phi$ defined by (33), we have

$$
b_{0}^{2} \int_{m}^{\infty} \frac{d^{2 x}}{F^{2}\left(b_{0} d^{x}\right)} d x \leq \phi(m) \leq b_{0}^{2} \int_{m-1}^{\infty} \frac{d^{2 x}}{F^{2}\left(b_{0} d^{x}\right)} d x,
$$

where we make use of $z / F(z)$ strictly decreasing for $z$ large. The change of variables $y=b_{0} d^{x}$ yields

$$
\frac{1}{\ln (d)} \int_{b_{0} d^{m}}^{\infty} \frac{y}{F^{2}(y)} d y \leq \phi(m) \leq \frac{1}{\ln (d)} \int_{b_{0} d^{m-1}}^{\infty} \frac{y}{F^{2}(y)} d y .
$$

Now $\psi=b \circ \phi^{-1}$, so that $\psi^{-1}=\phi \circ b^{-1}$, that is, $\psi^{-1}(p)=\phi\left(\log _{d}\left(p / b_{0}\right)\right)$. In conjunction with (36) this yields bounds

$$
\frac{1}{\ln (d)} \int_{p}^{\infty} \frac{y}{F^{2}(y)} d y \leq \psi^{-1}(p) \leq \frac{1}{\ln (d)} \int_{p / d}^{\infty} \frac{y}{F^{2}(y)} d y .
$$

Lemma B.1 of Appendix B asserts the existence of constants $C_{1}$ and $0<\beta \leq 1$ (dependent upon $F$ and $d$, of course) such that

$$
\int_{p / d}^{\infty} \frac{y}{F^{2}(y)} d y \leq C_{1}\left(\frac{p^{2}}{F^{2}(p)}\right)^{\beta} \text { for all } p>1,
$$


which upon combination with the last estimate of (37) gives the upper bound

$$
\psi^{-1}(p) \leq \frac{C_{1}}{\ln (d)}\left(\frac{p^{2}}{F^{2}(p)}\right)^{\beta} .
$$

The constant $C_{1}$ may be taken arbitrarily close to $\frac{1}{2 \delta_{1}}$, where $\delta_{1}$ is the constant from the superlinearity assumption (A2). If we define $G(z)=F(z) / z$ and $L(z)=\frac{C_{1}}{\ln (d)} \frac{1}{z^{2 \beta}}$, then inequality (38) can be written $\psi^{-1}(p) \leq L(G(p))$ for $p$ sufficiently large. Since $\psi$ is strictly decreasing in its argument, and since $L(G(p)) \rightarrow 0$ as $p \rightarrow \infty$ (note that $L(G(p)$ ) is also strictly decreasing in $p)$, we conclude that $\psi(z) \leq G^{-1}\left(L^{-1}(z)\right)$ for $z$ sufficiently close to zero. This yields

$$
\psi(z) \leq G^{-1}\left(\frac{C_{2}}{(\sqrt{z})^{1 / \beta}}\right)
$$

where $C_{2}=\left(\frac{C_{1}}{\ln (d)}\right)^{1 /(2 \beta)}$. In combination with (35) this gives

$$
u\left(t^{*}-\epsilon\right) \leq d G^{-1}\left(\frac{C_{2} D^{1 /(2 \beta)} d^{1 / \beta}}{2^{1 / \beta}(\sqrt{\epsilon})^{1 / \beta}}\right)
$$

or

$$
u(t) \leq d G^{-1}\left(\frac{C_{2} D^{1 /(2 \beta)} d^{1 / \beta}}{2^{1 / \beta}\left(\sqrt{t^{*}-t}\right)^{1 / \beta}}\right) .
$$

This is exactly an estimate of the type asserted in the statement of this proposition, with $\gamma=1 / \beta>1, K=d>1$ and $C=\frac{\left(C_{1} D\right)^{1 /(2 \beta)} d^{1 / \beta}}{(\ln d)^{1 /(2 \beta)} 2^{1 / \beta}}$. As $d$ and $D$ approach $1, \gamma$ approaches $1, K$ approaches 1 and $C$ approaches $\frac{C_{1}^{1 / 2}}{2(\ln d)^{1 / 2}}$, which may be picked arbitrarily close to $\frac{1}{2 \sqrt{2}\left(\delta_{1} \ln d\right)^{1 / 2}}$. These last observations all follow from Remark 8 of Appendix B.

REMARK 5. As an example, we may consider the function $F(u)=|u|^{p-1} u+u$ for $p>1$. In this case $G(u)=u^{p-1}+1, u>0$, so that $G^{-1}(z)=(z-1)^{1 /(p-1)} \leq C^{\prime} / z^{1 /(p-1)}$ for any $C^{\prime}>C$ and $z$ sufficiently large. The upper bound on $u_{1}(t)$ then becomes

$$
u_{1}(t) \leq K^{\prime}\left(t^{*}-t\right)^{-\gamma /(2(p-1))}
$$

for a suitable constant $K^{\prime}$. This bound is (modulo $\gamma$ ) of the same form as the lower bound in Remark 4

As another example, consider $F(u)=\sinh (u)$. Here $G(u)=\sinh (u) / u$ and $G^{-1}(z)=$ $\ln (z)+o(\ln (z))$ as $z \rightarrow \infty$. We then obtain the bound

$$
u_{1}(t) \leq-\frac{\gamma K}{2} \ln \left(t^{*}-t\right)+O\left(\ln \left|\ln \left(t^{*}-t\right)\right|\right),
$$

where $\gamma$ and $K$ can be taken arbitrarily close to 1 .

Remark 6. For the validity of Propositions 4.2 and 4.3 it is irrelevant whether $F$ is odd and whether $F^{\prime}(0)=1$. More precisely, the assumption (A1) may there be replaced by the condition that $F \in C^{2}(\mathbb{R})$, with $F, F^{\prime}$, and $F^{\prime \prime}$ being positive for a sufficiently large argument. 
4.3. Boundedness of the solution in the interior. In this section we show that for each fixed $x \in(0,1)$ the solution $u(x, t)$ remains bounded for $0 \leq t<t^{*}$. We suppose that $u_{0}$ and $u_{1}$ are both defined on $\left[0, t^{*}\right.$ ), and that $u_{1}$ (or for that matter, both $u_{1}$ and $u_{0}$ ) blows up at $t=t^{*}$ in a manner controlled by the estimate in Proposition 4.3 . As per Remark 3 we may assume that $u_{1}$ grows monotonically as $t \rightarrow t^{*}$. According to (7), the strictly increasing function $G(u)=F(u) / u$ satisfies $c u^{\delta_{1}} \leq G(u)$ for $u \geq 0$, where $\delta_{1}>0$ is the constant from the superlinearity condition (A2), so that $G^{-1}(u) \leq \tilde{c} u^{1 / \delta_{1}}$ for some constant $\tilde{c}$. The upper bound from Proposition 4.3 then guarantees that for some constant $C$,

$$
u_{1}(t) \leq C /\left(\sqrt{t^{*}-t}\right)^{\gamma / \delta_{1}} \text { for } t \text { close to } t^{*}
$$

where $\gamma$ can be chosen arbitrarily close to 1 .

We also need a bound on $F\left(u_{1}(t)\right)$, and so we shall introduce one additional assumption on $F$, namely that for fixed $K>1$, sufficiently close to 1 ,

there exist constants $C_{K}, A_{K}$ and $M_{K}$ such that

$$
F(K u) \leq C_{K} F(u)^{A_{k}} \text { for } u>M_{K} .
$$

This condition is, in some sense, another type of exponential bound on the growth of $F$, and is easily checked for any specific function; e.g., it holds for $F(x)=|x|^{p-1} x+x$ or $F(x)=\sinh (a x)$. With $K>1$ being the constant from Proposition 4.3 we have

$$
\begin{aligned}
F\left(u_{1}(t)\right) & =u_{1}(t) G\left(u_{1}(t)\right) \\
& \leq u_{1}(t) G\left(K G^{-1}\left(\frac{C}{\left(t^{*}-t\right)^{\gamma / 2}}\right)\right) .
\end{aligned}
$$

Note that $K$ may be chosen arbitrarily close to 1 . We thus also have, for any $z \geq M_{K}$,

$$
\begin{aligned}
G\left(K G^{-1}(z)\right) & =\frac{F\left(K G^{-1}(z)\right)}{K G^{-1}(z)} \\
& \leq C_{K} \frac{\left(F\left(G^{-1}(z)\right)\right)^{A_{K}}}{K G^{-1}(z)} \\
& =\tilde{C}_{K} z^{A_{k}}\left(G^{-1}(z)\right)^{A_{K}-1}
\end{aligned}
$$

with $\tilde{C}_{K}=C_{K} / K$. Here we make use of the assumption (A4) and the fact that $F\left(G^{-1}(z)\right)=z G^{-1}(z)$. If we combine the estimates (40), (41), and (39) (using $z=$ $\left.C /\left(\sqrt{t^{*}-t}\right)^{\gamma}\right)$ we obtain

$$
F\left(u_{1}(t)\right) \leq \frac{\tilde{C}}{\left(\sqrt{t^{*}-t}\right)^{A_{K} \gamma\left(1+1 / \delta_{1}\right)}} .
$$


From (11) we know that $u(x, t), 0<x<1,0<t<t^{*}$ may be represented as

$$
\begin{aligned}
u(x, t)= & \frac{1}{2 \sqrt{\pi t}} \int_{0}^{1} e^{-\frac{(x-y)^{2}}{4 t}} f(y) d y+\frac{1}{2} \int_{0}^{t} \frac{e^{-\frac{(x-1)^{2}}{4(t-s)}}}{\sqrt{\pi(t-s)}} F\left(u_{1}(s)\right) d s \\
& +\frac{1}{2} \int_{0}^{t} \frac{e^{-\frac{x^{2}}{4(t-s)}}}{\sqrt{\pi(t-s)}} F\left(u_{0}(s)\right) d s+x \int_{0}^{t} \frac{e^{-\frac{x^{2}}{4(t-s)}}}{4 \sqrt{\pi}(t-s)^{3 / 2}} u_{0}(s) d s \\
& +(1-x) \int_{0}^{t} \frac{e^{-\frac{(x-1)^{2}}{4(t-s)}}}{4 \sqrt{\pi}(t-s)^{3 / 2}} u_{1}(s) d s
\end{aligned}
$$

It is clear that the integral involving the initial condition $f$ stays bounded (in any norm) as $t$ approaches $t^{*}$. The estimates (39) and (42) show that $u_{1}(s)$ and $F\left(u_{1}(s)\right)$ grow at most like a negative power of $t^{*}-s$ as $s \rightarrow t^{*}$. At any fixed interior point $0<x_{0}<1$ the kernels in the corresponding two integrals (and all their derivatives) behave like $e^{-c /(t-s)}$ for $s$ near $t$, with $c>0$. For this reason it follows immediately that the integrals involving $u_{1}(s)$ and $F\left(u_{1}(s)\right)$ stay bounded as $t \rightarrow t^{*}$ for any fixed $x=x_{0} \in(0,1)$. The same argument applies to the integrals involving $u_{0}(s)$ and $F\left(u_{0}(s)\right)$ if $u_{0}$ blows up in a matter controlled by the estimate of Proposition 4.3. In summary we have proven:

Under the additional assumption (A4), the solution $u\left(x_{0}, t\right)$ stays bounded (in any norm) in a neighborhood of any fixed interior point $x_{0} \in(0,1)$, as $t \rightarrow t^{*}$.

Appendix A. A monotonicity result. The goal of this section is to give a proof of Proposition 4.1. Before we do so, it will be useful to establish the following two closely related lemmata.

Lemma A.1. Let $\phi(t)$ be a function in $C^{\alpha}\left[0, t^{*}\right)$ for some $\alpha>1 / 2,0<t^{*}<\infty$, and suppose $\phi$ satisfies an integral equation of the form

$$
P(\phi(t))=\int_{0}^{t} \frac{H(s, \phi(s))}{\sqrt{t-s}} d s+q(t),
$$

where $P \in C^{1}(-\infty, \infty)$ is strictly increasing, $q \in C^{1}\left[0, t^{*}\right]$, and $H \in C^{1}\left(\left[0, t^{*}\right) \times\right.$ $(-\infty, \infty))$. Assume that $H(s, z)$ is nondecreasing in each argument and that

$$
\lim _{z \rightarrow \infty} H(s, z)=\infty
$$

for each fixed $s \in\left[0, t^{*}\right)$. Suppose additionally that $\lim \sup _{t \rightarrow t^{*}} \phi(t)=\infty$. For $R \in$ $(\inf \phi, \infty)$, let $t_{R} \in\left[0, t^{*}\right)$ be defined as

$$
t_{R}=\inf \left\{t \in\left[0, t^{*}\right): \phi(t)=R\right\} .
$$

Then for any sufficiently large $R$ there exists $h_{R}>0$ so that

$$
\phi\left(t_{R}\right)<\phi\left(t_{R}+h\right) \text { for } 0<h \leq h_{R} .
$$

We may use a common value $h_{R}=h_{I}^{*}>0$ for all $R$ (sufficiently large but) in a bounded interval $I$. 
Proof. For convenience define an operator $Q$ as

$$
Q(\phi)(t)=\int_{0}^{t} \frac{H(s, \phi(s))}{\sqrt{t-s}} d s+q(t) .
$$

Given that the function $P$ is strictly increasing we can establish the lemma by showing that $Q(\phi)\left(t_{R}+h\right)-Q(\phi)\left(t_{R}\right)>0$ when $h>0$, that is,

$$
\begin{aligned}
0< & \int_{0}^{t_{R}+h} \frac{H(s, \phi(s))}{\sqrt{t_{R}+h-s}} d s+q\left(t_{R}+h\right)-\int_{0}^{t_{R}} \frac{H(s, \phi(s))}{\sqrt{t_{R}-s}} d s-q\left(t_{R}\right) \\
= & \int_{t_{R}}^{t_{R}+h} \frac{H(s, \phi(s))}{\sqrt{t_{R}+h-s}} d s+\int_{0}^{t_{R}} H(s, \phi(s))\left(\frac{1}{\sqrt{t_{R}+h-s}}-\frac{1}{\sqrt{t_{R}-s}}\right) d s \\
& \quad+q\left(t_{R}+h\right)-q\left(t_{R}\right) .
\end{aligned}
$$

This inequality can be written as

$$
\begin{aligned}
& \int_{0}^{t_{R}} H(s, \phi(s))\left(\frac{1}{\sqrt{t_{R}-s}}-\frac{1}{\sqrt{t_{R}+h-s}}\right) d s+q\left(t_{R}\right)-q\left(t_{R}+h\right) \\
& \quad<\int_{t_{R}}^{t_{R}+h} \frac{H(s, \phi(s))}{\sqrt{t_{R}+h-s}} d s .
\end{aligned}
$$

Since $q \in C^{1}\left[0, t^{*}\right]$ we have $\left|q\left(t_{R}\right)-q\left(t_{R}+h\right)\right| \leq C_{q} h$ for some constant $C_{q}$ independent of $t_{R}$ and $h$. Inequality (43), and hence $Q(\phi)\left(t_{R}+h\right)-Q(\phi)\left(t_{R}\right)>0$, will hold if we obtain

$$
\int_{0}^{t_{R}} H(s, \phi(s))\left(\frac{1}{\sqrt{t_{R}-s}}-\frac{1}{\sqrt{t_{R}+h-s}}\right) d s+C_{q} h<\int_{t_{R}}^{t_{R}+h} \frac{H(s, \phi(s))}{\sqrt{t_{R}+h-s}} d s,
$$

for $0<h<h_{R}$. We will establish an upper bound $I_{1}$ on the left side of (44), and a lower bound $I_{2}$ on the right side of (44), with $I_{1}<I_{2}$. This will establish (44) and the lemma.

We first obtain a lower bound for the integral on the right in (44). Let $R$ be any positive value in the interval (inf $\phi, \infty)$, and let $t_{R}$ be as in the statement of the lemma. For some constant $C_{\alpha, R}$ we have

$$
\frac{\left|\phi(t)-\phi\left(t_{R}\right)\right|}{\left|t-t_{R}\right|^{\alpha}} \leq C_{\alpha, R},
$$

for all $t$ in a neighborhood of $t_{R}$. The constant $C_{\alpha, R}$ is uniformly bounded for $R$ in any compact subinterval of (inf $\phi, \infty)$. In particular, for all sufficiently small $h$ we have

$$
\left|\phi\left(t_{R}+h\right)-R\right| \leq C_{\alpha, R} h^{\alpha},
$$

since $\phi\left(t_{R}\right)=R$. As a consequence, $\phi\left(t_{R}+h\right) \geq R-C_{\alpha, R} h^{\alpha}$, and for $s \in\left[t_{R}, t_{R}+h\right]$ we have

$$
\phi(s) \geq R-C_{\alpha, R} h^{\alpha} .
$$

For sufficiently small $h$ the integral on the right side in (44) is then bounded below by

$$
\begin{aligned}
\int_{t_{R}}^{t_{R}+h} \frac{H(s, \phi(s))}{\sqrt{t_{R}+h-s}} d s & \geq \int_{t_{R}}^{t_{R}+h} \frac{H\left(t_{R}, R-C_{\alpha, R} h^{\alpha}\right)}{\sqrt{t_{R}+h-s}} d s \\
& =2 \sqrt{h} H\left(t_{R}, R-C_{\alpha, R} h^{\alpha}\right) .
\end{aligned}
$$


Here we have made use of the fact that $H$ is nondecreasing in each argument. Since $H$ is $C^{1}$ we find that

$$
\int_{t_{R}}^{t_{R}+h} \frac{H(s, \phi(s))}{\sqrt{t_{R}+h-s}} d s \geq 2 \sqrt{h}\left[H\left(t_{R}, R\right)-\tilde{C}_{\alpha, R} h^{\alpha}\right]
$$

for some constant $\tilde{C}_{\alpha, R}$. The constant $\tilde{C}_{\alpha, R}$ is uniformly bounded for $R$ in any compact subinterval of $(\inf \phi, \infty)$. To obtain an upper bound on the integral on the left side in (44) we begin with

$$
\begin{aligned}
& \int_{0}^{t_{R}} H(s, \phi(s))\left(\frac{1}{\sqrt{t_{R}-s}}-\frac{1}{\sqrt{t_{R}+h-s}}\right) d s \\
& \quad \leq \int_{0}^{t_{R}} H\left(t_{R}, R\right)\left(\frac{1}{\sqrt{t_{R}-s}}-\frac{1}{\sqrt{t_{R}+h-s}}\right) d s \\
& \quad=2 H\left(t_{R}, R\right)\left(\sqrt{t_{R}}+\sqrt{h}-\sqrt{t_{R}+h}\right),
\end{aligned}
$$

where the above inequality follows from $\phi(t) \leq R$ for $t \leq t_{R}$ and the properties of $H$. It is easy to see that

$$
\sqrt{1+x}-1 \geq \frac{1}{4} x
$$

for all $0 \leq x \leq 8$. With $x=h / t$ this inequality becomes $\sqrt{1+h / t}-1 \geq \frac{h}{4 t}$ for $0 \leq h \leq 8 t$. Multiplication by $-2 \sqrt{t}$ and addition of $2 \sqrt{h}$ to both sides yields

$$
2(\sqrt{t}+\sqrt{h}-\sqrt{t+h}) \leq 2 \sqrt{h}-\frac{h}{2 \sqrt{t}}
$$

for $0 \leq h \leq 8 t$. By insertion of (47) into the right side of (46) we obtain the upper bound

$$
\int_{0}^{t_{R}} H(s, \phi(s))\left(\frac{1}{\sqrt{t_{R}-s}}-\frac{1}{\sqrt{t_{R}+h-s}}\right) d s \leq H\left(t_{R}, R\right)\left(2 \sqrt{h}-\frac{h}{2 \sqrt{t_{R}}}\right)
$$

for suitably small $h$. Here we use that $t_{R}$ is bounded away from 0 , for instance $t^{*} / 2<$ $t_{R}<t^{*}$, for $R$ sufficiently large. If we make use of the upper and lower bounds (48) and (45) we see that the inequality (44) (and thus $Q(\phi)\left(t_{R}+h\right)>Q(\phi)\left(t_{R}\right)$ ) will be established if

$$
C_{q} h+H\left(t_{R}, R\right)\left(2 \sqrt{h}-\frac{h}{2 \sqrt{t_{R}}}\right)<2 \sqrt{h}\left[H\left(t_{R}, R\right)-\tilde{C}_{\alpha, R} h^{\alpha}\right] .
$$

A cancelation and a bit of algebra show that (49) is equivalent to

$$
\left(\frac{H\left(t_{R}, R\right)}{2 \sqrt{t_{R}}}-C_{q}\right) h>2 \tilde{C}_{\alpha, R} h^{\alpha+1 / 2} .
$$

Due to the monotonicity of $H(\cdot, R)$, and since $0 \leq t_{R}<t^{*}$, we obtain (50) if we establish that for fixed $t_{1} \in\left[0, t^{*}\right)$,

$$
\left(\frac{H\left(t_{1}, R\right)}{2 \sqrt{t^{*}}}-C_{q}\right) h>2 \tilde{C}_{\alpha, R} h^{\alpha+1 / 2}
$$

for all $R$ sufficiently large (with $t_{R} \geq t_{1}$ ) and all $h$ sufficiently small. Given the properties of $H$, the coefficient of $h$ on the left side of (51) is clearly uniformly positive if $R$ is chosen sufficiently large. Since the right side of (51) is $O\left(h^{\beta}\right)$ with $\beta=\alpha+\frac{1}{2}$ we now see that (51) holds for all sufficiently small $h$. This establishes inequalities (50), (49), and (44), and 
the lemma. The fact that the constant $\tilde{C}_{\alpha, R}$ is uniformly bounded for $R$ in any compact subinterval of (inf $\phi, \infty)$ immediately implies that we may use a common $h_{R}=h_{I}^{*}$ for $R$ sufficiently large, but in a bounded interval $I$.

As a consequence of the previous lemma we obtain

Lemma A.2. Let the notation be as in Lemma A.1. There exists $R_{0}$ with the property that for any $R \geq R_{0}$ one may find $\tilde{h}_{R}>0$ so that $\phi$ is strictly increasing on the interval $\left[t_{R}, t_{R}+\tilde{h}_{R}\right]$.

Proof. For a given sufficiently large $R$, let $h_{R}$ be as asserted by Lemma A.1 Let $I$ be the compact interval $\phi\left(\left[t_{R}, t_{R}+h_{R}\right]\right)$, and let $\tilde{h}_{R}=h_{I} \leq h_{R}$ be the common increment that may be used for all values in $I$, according to Lemma A.1. We proceed to show that $\phi$ is strictly increasing on $\left[t_{R}, t_{R}+\tilde{h}_{R}\right]$. Suppose $t, \tilde{t} \in\left[t_{R}, t_{R}+\tilde{h}_{R}\right]$ with $t<\tilde{t}$. Define $T=\phi(t)$; since $\phi(s)<R=\phi\left(t_{R}\right) \leq \phi(t)$ for $s<t_{R}$ it follows immediately that $t_{T} \in\left[t_{R}, t_{R}+\tilde{h}_{R}\right]$. We also have $t_{T} \leq t<\tilde{t}$. From Lemma A.1, and the fact that the same $\tilde{h}_{R}$ may be used for all values in $I$ (and thus for $T$ ) it now follows that

$$
\phi\left(t_{T}\right)<\phi(s) \text { for all } s \in\left(t_{T}, t_{T}+\tilde{h}_{R}\right] .
$$

In particular, since $t_{T}<\tilde{t} \leq t_{R}+\tilde{h}_{R} \leq t_{T}+\tilde{h}_{R}$, it follows that

$$
\phi(t)=\phi\left(t_{T}\right)<\phi(\tilde{t}) \text {, }
$$

which completes the proof.

Using Lemma A.2 it is now fairly simple to give the proof of Proposition 4.1

Proof of Proposition 4.1. We shall show that $\phi$ is strictly increasing on the interval $\left[t_{R_{0}}, t^{*}\right)$, where $R_{0}$ and $t_{R_{0}}$ are as in Lemma A.2 To this end we define

$\tilde{t}^{*}=\sup \left\{t: t_{R_{0}} \leq t<t^{*}\right.$, and $\phi$ is strictly increasing on the interval $\left.\left[t_{R_{0}}, t\right]\right\}$.

It is clear that $\tilde{t}^{*} \leq t^{*}$, and from Lemma A.2 we know that $t_{R_{0}}<\tilde{t}^{*}$. The function $\phi$ is strictly increasing on the interval $\left[t_{R_{0}}, t^{*}\right)$ if and only if $\tilde{t}^{*}=t^{*}$. We proceed by contradiction: Suppose $t_{R_{0}}<\tilde{t}^{*}<t^{*}$. By continuity we know that $\phi$ is strictly increasing on the interval $\left[t_{R_{0}}, \tilde{t}^{*}\right]$. If we define $T=\phi\left(\tilde{t}^{*}\right)$, then we have $R_{0}<T$ and $t_{T}=\tilde{t}^{*}$, and so by Lemma A.2 we conclude that $\phi$ is strictly increasing on some interval $\left[t_{T}, t_{T}+h_{T}\right]=$ $\left[\tilde{t}^{*}, \tilde{t}^{*}+h_{T}\right], h_{T}>0$. By combination with the strict monotonicity on $\left[t_{R_{0}}, \tilde{t}^{*}\right]$ this yields that $\phi$ is strictly increasing on the interval $\left[t_{R_{0}}, \tilde{t}^{*}+h_{T}\right]$, in contradiction to the definition of $\tilde{t}^{*}$. We therefore conclude that $\tilde{t}^{*}=t^{*}$, and this completes the proof of Proposition 4.1

REMARK 7. We note that a result entirely similar to that of Proposition 4.1 holds if $H$ is nonincreasing (in $z$ ) with $\lim _{z \rightarrow-\infty} H(s, z)=\infty, P$ is strictly decreasing, and $\lim \sup _{t \rightarrow t^{*}} \phi(t)=-\infty$. The appropriate conclusion is then that $\phi(t)$ is strictly decreasing on some interval $\left[t_{R}, t^{*}\right)$ for $R$ sufficiently negative.

Appendix B. An integral estimate. In this appendix we establish the following estimate, which was used for the verification of the upper bound in Section 4.2 . 
Lemma B.1. Let $F \in C^{2}(\mathbb{R})$ with $F(u)>0, u>0$, be superlinear in the sense of (A2) and suppose that $F^{\prime}(u) / F(u)>0$ is nonincreasing for $u \geq M>0$ as required by assumption (A3). For any $d>1$ there exist constants $0<\beta \leq 1$ and $C$ such that

$$
\int_{p / d}^{\infty} \frac{y}{F^{2}(y)} d y \leq C\left(\frac{p^{2}}{F^{2}(p)}\right)^{\beta} \text { for all } p>1 .
$$

Proof. As noted in Section 2, the condition (A2) together with the fact that $F(u)>0$ for $u>0$ implies that $F(u) / u^{1+\delta}, u \geq M$, is strictly increasing for any $\delta<\delta_{1}$. Therefore

$$
\frac{F(y)}{y^{1+\delta}} \geq \frac{F(p / d)}{(p / d)^{1+\delta}} \text { for } y \geq p / d \geq M,
$$

so that $F(y) \geq \frac{F(p / d)}{(p / d)^{1+\delta}} y^{1+\delta}$, or

$$
\frac{1}{F^{2}(y)} \leq \frac{(p / d)^{2(1+\delta)}}{F^{2}(p / d)} \frac{1}{y^{2(1+\delta)}}
$$

As a result,

$$
\begin{aligned}
\int_{p / d}^{\infty} \frac{y}{F^{2}(y)} d y & \leq \frac{(p / d)^{2(1+\delta)}}{F^{2}(p / d)} \int_{p / d}^{\infty} y^{-1-2 \delta} d y \\
& =\frac{p^{2+2 \delta}}{2 \delta d^{2+2 \delta} F^{2}(p / d)}\left(\frac{d}{p}\right)^{2 \delta} \\
& =\frac{p^{2}}{2 \delta d^{2} F^{2}(p / d)}, \quad p / d \geq M .
\end{aligned}
$$

We thus have

$$
\begin{aligned}
\limsup _{p \rightarrow \infty}\left(\frac{F^{2}(p)}{p^{2}}\right)^{\beta} \int_{p / d}^{\infty} \frac{y}{F^{2}(y)} d y & \leq \limsup _{p \rightarrow \infty}\left(\frac{F^{2}(p)}{p^{2}}\right)^{\beta} \frac{p^{2}}{2 \delta d^{2} F^{2}(p / d)} \\
& =\frac{1}{2 \delta d^{2}} \limsup _{p \rightarrow \infty} p^{2(1-\beta)} \frac{F^{2 \beta}(p)}{F^{2}(p / d)} .
\end{aligned}
$$

We shall now show that the limsup on the right in (53) is finite. Since the expression

$$
\left(\frac{F^{2}(p)}{p^{2}}\right)^{\beta} \int_{p / d}^{\infty} \frac{y}{F^{2}(y)} d y
$$

is bounded for $p$ in any bounded interval $(1, N)$, the boundedness of the lim sup is sufficient to verify the estimate of this lemma. Let $H(u)=F^{\prime}(u) / F(u)$. For a fixed $d>1$ choose

$$
\beta=\frac{K_{1}-1}{d K_{1}-1},
$$

where $K_{1}=1+\delta_{1}>1$ and $\delta_{1}$ is the constant in the superlinearity assumption (A2). Clearly $0<\beta<1$. Note that this choice for $\beta$ yields

$$
\frac{1}{d \beta}-\frac{1-\beta}{d K_{1} \beta}=1 \text {. }
$$


Since $H(u), u \geq M$ is positive and nonincreasing, we have $H(u) \leq H(u / d)$ for $u \geq M d$, and so with $\beta$ given by (54),

$$
\begin{aligned}
\frac{H(u)}{H(u / d)} & \leq 1 \\
& =\frac{1}{d \beta}-\frac{1-\beta}{d K_{1} \beta} \\
& =\frac{1}{d \beta}-\frac{1-\beta}{\beta u} \frac{1}{d K_{1} / u} \\
& \leq \frac{1}{d \beta}-\frac{1-\beta}{\beta u} \frac{1}{H(u / d)},
\end{aligned}
$$

for $u \geq M d$. The last inequality in (55) follows from (A2) in the form $H(u / d) \geq d K_{1} / u$. We multiply both sides of (55) by $\beta H(u / d)$ to obtain

$$
\beta H(u) \leq \frac{1}{d} H(u / d)-\frac{1-\beta}{u} \text { for } u \geq M d .
$$

Integration of both sides of (56) from $u=M d$ to $u=p$ (note that $H(u)=F^{\prime}(u) / F(u)$ ) and simplification yields

$$
F^{\beta}(p) \leq \tilde{C} F(p / d) p^{\beta-1}
$$

with $\tilde{C}=\frac{F^{\beta}(M d)(M d)^{1-\beta}}{F(M)}$. The boundedness of the limsup in (153) follows, and this proves the lemma.

REMARK 8. Note that with $\beta$ given by (54), $\beta$ will approach 1 as $d$ approaches 1 . Moreover, the constant $\tilde{C}$ in the proof of Lemma B.1 also approaches 1 as $d \rightarrow 1$. The constant $C$ in the estimate (52) can be taken as

$$
C=\frac{1}{2 \delta d^{2}} \tilde{C}^{2}=\frac{F^{2 \beta}(M d) M^{2(1-\beta)}}{2 \delta d^{2 \beta} F^{2}(M)},
$$

where $\beta$ is given by (54). As a consequence, a possible constant $C$ that may be used in (52) will approach $\frac{1}{2 \delta}$ as $d \rightarrow 1$. Here $\delta$ may be picked arbitrarily close to $\delta_{1}$, the constant from the superlinearity assumption (A2).

Acknowledgments. The research of M.S. Vogelius was partially supported by NSF grant DMS-0604999.

\section{REFERENCES}

[1] C. Bandle and H. Brunner, Blowup in diffusion equations: A survey, Journal of Computational and Applied Mathematics, 97, 1998, pp. 3-22. MR1651764 (99g:35061)

[2] K. Bryan and M.S. Vogelius, Transient behavior of solutions to a class of nonlinear boundary value problems, to appear in the Quarterly of Applied Math.

[3] K. Deng, The blow-up behavior of the heat equation with Neumann boundary conditions, J. Math. Anal. Appl., 188, 1994, pp. 641-650. MR1305473 (95i:35120)

[4] K. Deng and M. Xu, Remarks on blow-up behavior for a nonlinear diffusion equation with Neumann boundary conditions, Proc. Amer. Math. Soc., 127, 1999, pp. 167-172. MR1485467 (99b:35102)

[5] M. Fila and J. Filo, Blow-up on the boundary: A survey, pp. 67-78 in Singularities and Differential Equations, S. Janeczko et al. (eds), Banach Center Publ. 33, Polish Acad. Sciences, Warsaw, 1996. MR.1449147 (98c:35076)

[6] M. Fila and J. Guo, Complete blow-up and incomplete quenching for the heat equation with a nonlinear boundary condition, Nonlinear Analysis, 48, 2002, pp. 995-1002. MR1880259 
[7] S. Fu, J. Guo and J. Tsai Blow-up behavior for a semilinear heat equation with a nonlinear boundary condition, Tohoku Math. J. (2), 55, 2003, pp. 565-581. MR2017226 (2004h:35112)

[8] S. Kichenassamy, Recent Progress on Boundary Blow-up, pp. 329-341 in "Elliptic and Parabolic Problems", Volume 63 of the Book Series "Progress in Nonlinear Differential Equations and Their Applications", Birkhäuser, Basel, 2005. MR2176725 (2006e:35022)

[9] H. Levine and L. Payne, Nonexistence theorems for the heat equation with nonlinear boundary conditions and for the porous medium equation backward in time, J. Differential Equations, 16, 1974, pp. 319-334. MR0470481 (57:10235)

[10] Z. Lin and M. Wang, The blow-up properties of solutions to semilinear heat equations with nonlinear boundary conditions, Z. Angew. Math. Phys., 50, 1999, pp. 361-374. MR.1697712 (2000c:35132)

[11] F. Quiros, J. D. Rossi and J. L. Vazquez, Complete blow-up and thermal avalanche for heat equations with nonlinear boundary conditions, Comm. in PDE, 27, 2002, pp. 395-424. MR1886965 (2002k:35041)

[12] C. Roberts, Recent results on blow-up and quenching for nonlinear Volterra equations, J. Comput. Applied Math., 205, 2007, pp. 736-743. MR2329649 (2008c:45006)

[13] W. Walter, On existence and nonexistence in the large of solutions of parabolic differential equations with a nonlinear boundary condition, SIAM J. Math. Anal., 6, 1975, pp. 85-90. MR0364868 $(51: 1122)$ 